\title{
Dexamethasone Protects Against Tourniquet-Induced Acute Ischemia-Reperfusion Injury in Mouse Hindlimb
}

\author{
Ryan M. Corrick ${ }^{\dagger}$, Huiyin Tu ${ }^{\dagger}$, Dongze Zhang ${ }^{\dagger}$, Aaron N. Barksdale, Robert L. Muelleman, \\ Michael C. Wadman and Yu-Long Li*
}

Department of Emergency Medicine, University of Nebraska Medical Center, Omaha, NE, United States

\section{OPEN ACCESS}

Edited by:

Susan V. Brooks,

University of Michigan, United States

Reviewed by:

Jian Xu,

University of Oklahoma Health

Sciences Center, United States

Kunihiro Sakuma,

Tokyo Institute of Technology, Japan

*Correspondence:

Yu-Long Li

yulongli@unmc.edu

†These authors have contributed equally to this work.

Specialty section: This article was submitted to

Striated Muscle Physiology, a section of the journa

Frontiers in Physiology

Received: 14 January 2018 Accepted: 06 March 2018

Published: 20 March 2018

Citation:

Corrick RM, Tu H, Zhang $D$, Barksdale AN, Muelleman RL,

Wadman MC and Li Y-L (2018)

Dexamethasone Protects Against

Tourniquet-Induced Acute

Ischemia-Reperfusion Injury in Mouse

Hindlimb. Front. Physiol. 9:244

doi: 10.3389/fphys.2018.00244
Extremity injuries with hemorrhage have been a significant cause of death in civilian medicine and on the battlefield. The use of a tourniquet as an intervention is necessary for treatment to an injured limb; however, the tourniquet and subsequent release results in serious acute ischemia-reperfusion (IR) injury in the skeletal muscle and neuromuscular junction (NMJ). Much evidence demonstrates that inflammation is an important factor to cause acute IR injury. To find effective therapeutic interventions for tourniquet-induced acute IR injuries, our current study investigated effect of dexamethasone, an anti-inflammatory drug, on tourniquet-induced acute IR injury in mouse hindlimb. In C57/BL6 mice, a tourniquet was placed on unilateral hindlimb (left hindlimb) at the hip joint for $3 \mathrm{~h}$, and then released for $24 \mathrm{~h}$ to induce IR. Three hours of tourniquet and $24 \mathrm{~h}$ of release (24-h IR) caused gastrocnemius muscle injuries including rupture of the muscle sarcolemma and necrosis $(42.8 \pm 2.3 \%$ for infarct size of the gastrocnemius muscle). In the NMJ, motor nerve terminals disappeared, and endplate potentials were undetectable in 24-h IR mice. There was no gastrocnemius muscle contraction in 24-h IR mice. Western blot data showed that inflammatory cytokines (TNF $\alpha$ and IL-1 $\beta$ ) were increased in the gastrocnemius muscle after 24-h IR. Treatment with dexamethasone at the beginning of reperfusion (1 mg/ $\mathrm{kg}$, i.p.) significantly inhibited expression of TNF $\alpha$ and IL-1 $\beta$, reduced rupture of the muscle sarcolemma and infarct size $(24.8 \pm 2.0 \%)$, and improved direct muscle stimulation-induced gastrocnemius muscle contraction in 24-h IR mice. However, this anti-inflammatory drug did not improve NMJ morphology and function, and sciatic nerve-stimulated skeletal muscle contraction in 24-h IR mice. The data suggest that one-time treatment with dexamethasone at the beginning of reperfusion only reduced structural and functional impairments of the skeletal muscle but not the NMJ through inhibiting inflammatory cytokines.

Keywords: tourniquet, acute ischemia-reperfusion injury, skeletal muscle, neuromuscular junction, cytokine, dexamethasone 


\section{INTRODUCTION}

Based on the data from the Center for Disease Control and Prevention, over 14 million persons with extremity injuries visit emergency rooms, with an estimated cost of $\$ 80$ billion each year (Center for Disease Control and Prevention, 2017). Exsanguinating injury of the extremities is a key factor of trauma fatalities in civilian medicine and on the battlefield (Mabry, 2006; Inaba et al., 2015; Ode et al., 2015). Tourniquet use is a primary tool in first-line treatment of severe limb hemorrhage (Mabry, 2006; Beekley et al., 2008; Kragh et al., 2012; Welling et al., 2012). The tourniquet is also used to create an optimal operating fields with bloodless in vascular surgeries and orthopedic, musculoskeletal reconstructive procedures (an estimated 15,000 procedures daily in the US and elsewhere) (Tai et al., 2011; Halladin et al., 2014). However, acute ischemia-reperfusion (IR) injuries induced by a tourniquet use with subsequent reperfusion usually occur, which include tissue apoptosis and necrosis in the skeletal muscle, and severe structural and functional damage in the neuromuscular junction (NMJ) (Aho et al., 1983; Mohler et al., 1999; Schoen et al., 2007; Tran et al., 2011, 2012; Gillani et al., 2012). These acute injuries also affect long-term recovery of NMJ function and skeletal muscle contraction from IR injuries, even resulting in complete limb paralysis as well as the need for secondary amputation (Kam et al., 2001; Noordin et al., 2009; Vignaud et al., 2010). These complications have led to the deemphasis and limitations of tourniquet use (Clasper et al., 2009; Kue et al., 2015).

Until now the cellular and molecular mechanisms responsible limb acute IR injuries are unclear. Some studies demonstrated that overproduction of inflammatory cytokines and reactive oxygen species in injured skeletal muscles during the acute IR causes neuronal damage and deficit of skeletal muscle function (Seekamp et al., 1993; Clementsen and Reikeras, 2008; Chen and Nunez, 2010; Eltzschig and Eckle, 2011). Based on this fact, inhibition of production and release of inflammatory cytokines is likely considered as one of the strategies for limb acute IR injury. Dexamethasone (Dex), a synthetic glucocorticoid and potent anti-inflammatory drug, is used clinically to treat many inflammatory and autoimmune diseases, including asthma, rheumatoid arthritis, lupus nephritis, inflammatory shock, etc. (Barnes, 2011). Therefore, we hypothesized that Dex could protect the limb from acute IR injury through inhibiting inflammatory cytokines because glucocorticoids reduce production and release of inflammatory cytokines in whole blood (Schwiebert et al., 1996; Smits et al., 1998; Schuld et al., 2001).

In general, both innervated motor nerve-muscle transmission (i.e., NMJ) and skeletal muscle are involved in skeletal muscle contractile function (Chervu et al., 1989; Fish et al., 1989; Eastlack et al., 2004). In this study, therefore, three purposes were performed: (1) to assess the morphological and functional changes of the skeletal muscle and NMJ; (2) to test whether Dex could attenuate the structural and functional impairment of the skeletal muscle and NMJ; (3) to measure the inhibitory effect of Dex on inflammatory cytokines in a murine model of tourniquetinduced 3-h ischemia and subsequent 24 -h reperfusion.

\section{MATERIALS AND METHODS}

\section{Animals}

Totally 63 male C57/BL6 mice (21 sham, 21 tourniquet-induced IR, and 21 tourniquet-induced IR + Dex mice) weighing 22-25g (Charles River Laboratory, Wilmington, MA) were used in this study. The mice were housed under controlled temperature and humidity and a 12:12-h dark-light cycle, and were provided water and mouse chow ad libitum. All experimental procedures were approved by the University of Nebraska Medical Center Institutional Animal Care and Use Committee and were carried out in accordance with the National Institutes of Health (NIH Publication No. 85-23, revised 1996) and the American Physiological Society's "Guides for the Care and Use of Laboratory Animals."

\section{A Mouse Model of Tourniquet-Induced Hindlimb IR and Drug Treatment}

Tourniquet-induced mouse hindlimb IR is a mature animal model and has been used in our previous studies (Tran et al., 2011, 2012; Tu et al., 2017; Zhang et al., 2017). Briefly, under anesthesia with a cocktail consisting of $100 \mathrm{mg} / \mathrm{kg}$ ketamine and $10 \mathrm{mg} / \mathrm{kg}$ xylazine, given as an intraperitoneal injection (10 $\mathrm{ml} / \mathrm{kg}$ body weight), the tourniquet in unilateral hindlimb (left hindlimb) was done for $3 \mathrm{~h}$ by setting an orthodontic rubber band at the hip joint, using a McGivney hemorrhoidal ligatorer. Then the orthodontic rubber band was released to begin reperfusion for $24 \mathrm{~h}$. During the period of this procedure, the level of anesthesia was continuously monitored by testing the respiratory patterns and toe pinch reflex. A heating pad was used to maintain body temperature at $37^{\circ} \mathrm{C}$ until the animals woke up. Our previous study has verified skeletal muscle IR in this animal model through testing blood flow in IR gastrocnemius muscles (Tran et al., 2011). Before waking up, mice were kept well hydrated with an intraperitoneal injection of $0.2 \mathrm{ml}$ normal saline every $2 \mathrm{~h}$.

Usually the contralateral skeletal muscle is used as an uninjured control muscle in a unilateral tourniquet-induced IR model (Woitaske and McCarter, 1998; Walters et al., 2008; Lotfi et al., 2013). However, it is possible that metabolites from injured skeletal muscles and drug treatments are also distributed into contralateral NMJs and skeletal muscles, affecting their functions. A sham group of mice was designed as the control group in the present study to avoid this possibility. The same procedure except for the application of the orthodontic rubber band was performed in sham-operated animals.

Dex (Sigma-Aldrich, St. Louis, MO) was dissolved in $0.9 \%$ $\mathrm{NaCl}$. IR mice were randomly divided into two groups for administration of normal saline or Dex $(1 \mathrm{mg} / \mathrm{kg})$, respectively. Normal saline solution or Dex $(0.2 \mathrm{ml})$ was intraperitoneally administered at the beginning of reperfusion. All terminal experiments were performed on mice after $24 \mathrm{~h}$ of reperfusion.

\section{In-Situ Detection of the Gastrocnemius Muscle Contractile Force}

The gastrocnemius muscle contractile force was measured in five sham, five tourniquet-induced IR, and five tourniquet-induced 
IR + Dex mice as described previously (Tu et al., 2017; Zhang et al., 2017). Briefly, under anesthesia ( $800 \mathrm{mg} \mathrm{kg}^{-1}$ urethane and $40 \mathrm{mg} \mathrm{kg}^{-1}$ chloralose, a mixing solution of $20 \%$ urethane and $1 \%$ chloralose, $4 \mathrm{ml} \mathrm{kg}^{-1}$, i.p.), the mouse was kept in prone position and maintained at $37^{\circ} \mathrm{C}$. The isolated middle and distal ends of the left gastrocnemius muscle were moistened by warmed saline. The distal end of the gastrocnemius muscle was sutured and connected to a wide range force transducer (AD Instruments, Colorado Springs, CO). The left sciatic nerve was exposed through splitting the left biceps femoris muscle along muscle fibers. Maximal electrical tetanus stimulation $(10 \mathrm{~V}, 50 \mathrm{~Hz}, 0.1-\mathrm{ms}$ pulse, 5-s duration) was produced by a bipolar platinum electrode placed on the distal cut end of the sciatic nerve to induce tetanic contractile force of the gastrocnemius muscle. A similar procedure was performed for direct gastrocnemius muscle stimulation $(10-50 \mathrm{~V}, 50 \mathrm{~Hz}, 0.1$-ms pulse, 5 -s duration). Considering spatial variability for direct gastrocnemius muscle stimulation, we selected 4 areas of gastrocnemius muscles for this measurement and collected these results as the data for one measured muscle. PowerLab 8/30 Data Acquisition Systems with LabChart 7 (AD Instruments, Colorado Springs, CO, USA) were used to record and analyze the muscle contractile force.

\section{Measurement of Infarct Size in the Gastrocnemius Muscle (Tran et al., 2011)} Infarct size of the gastrocnemius muscle induced by tourniquetrelated IR was tested by triphenyl tetrazolium chloride assay. After measurement of the gastrocnemius muscle contractile force, mouse gastrocnemius muscle was quickly isolated and cut into $1.5 \mathrm{~mm}$ transverse slices. The cut slices were cleaned by cold saline solution, and subsequently incubated with $1 \%$ triphenyl tetrazolium chloride solution (Sigma-Aldrich, St. Louis, MO) at room temperature for $1 \mathrm{~h}$. Slice images were digitalized by a Canon camera. In the obtained muscle images, the muscle with a dark purple red color stain was defined as the viable muscle, and the muscle with a pale brown color stain was defined as the infarcted muscle. After the viable muscle and infarcted muscle were quantified by Adobe Photoshop CS5, the ratio (\%, wet weight/wet weight) of the infarcted muscle to total gastrocnemius muscle (viable plus infarcted muscles) was calculated as the infarct size.

\section{Histological Evaluation of Skeletal Muscle Damage and Inflammatory Infiltration}

In the first experiment, gastrocnemius muscles from three sham, three tourniquet-induced IR, and three tourniquet-induced IR + Dex mice were successively fixed in Methacarn solution (300 $\mathrm{ml}$ methanol, $150 \mathrm{ml}$ chloroform, and $50 \mathrm{ml}$ acetic acid) for $48 \mathrm{~h}$, and $60 \%$ ethanol for $72 \mathrm{~h}$. The fixed muscle was embedded into paraffin wax after routine processing, and then cut into 4 - $\mu \mathrm{m}$-thick longitudinal sections. After deparaffinization, sections were stained with hematoxylin and eosin (H\&E, SigmaAldrich, St. Louis, MO) for inflammatory infiltration and with Masson's trichrome (Sigma-Aldrich, St. Louis, MO) for skeletal muscle damage. Stained sections were captured by a bright-field microscope (Leica Microsystems Inc., Buffalo Grove, IL) with digital camera (Qimaging MicroPublisher 3.3RTV).

In the second experiment, gastrocnemius muscles from three sham, three tourniquet-induced IR, and three tourniquet-induced IR + Dex mice were postfixed with $4 \%$ paraformaldehyde for $12 \mathrm{~h}$, and then soaked in $30 \%$ sucrose for $12 \mathrm{~h}$ at $4^{\circ} \mathrm{C}$ for cryostat procedure. The muscle was cut into $10-\mu \mathrm{m}$-thick cross-sections in a freezing cryostat at $-20^{\circ} \mathrm{C}$. Tissue sections were processed with standard immunocytochemical staining procedure. Briefly, tissue sections were permeabilized with $0.3 \%$ Triton X-100 (Thermo Fisher Scientific, Waltham, MA) in PBS at room temperature for $20 \mathrm{~min}$. After tissue sections were successively incubated with $10 \%$ normal goat serum (Jackson Immunoresearch Labs Inc.), rabbit anti-laminin antibody (Sigma-Aldrich, St. Louis, MO), and goat anti-rabbit IgG labeled with Alexa Fluor 594 (Thermo Fisher Scientific, Waltham, MA), tissue images were obtained using a Leica fluorescent microscope with digital camera (Qimaging Retiga Exi Fast 1394).

\section{Electrophysiological Recording of the EPP In-situ}

The EPP recording was performed in five sham, five tourniquetinduced IR, and five tourniquet-induced IR + Dex mice as described previously (Tu et al., 2017; Zhang et al., 2017). Under anesthesia ( $800 \mathrm{mg} / \mathrm{kg}$ urethane and $40 \mathrm{mg} / \mathrm{kg}$ chloralose, i.p.), the isolated middle and distal end of the left gastrocnemius muscle was moistened by warmed saline solution. To avoid the influence of muscle contraction on the EPP recording, a specific muscle $\mathrm{Na}^{+}$channel blocker, $\mu$-conotoxin GIIIB $(4 \mu \mathrm{M}, 200 \mu \mathrm{L})$ was locally provided into the gastrocnemius muscle to inhibit muscle contraction. A glass microelectrode filled with $3 \mathrm{M} \mathrm{KCl}$ (5-15 $\mathrm{M} \Omega$ pipette resistance) was slowly inserted into the gastrocnemius muscle fiber and connected with an intracellular preamplifier (IX1; Dagan Corporation, Minneapolis, MN, USA) for the EPP recording. When miniature endplate potentials (mEPPs) could be recorded to determine an endplate close to the electrode, the EPPs were recorded by intracellular recording technique under the electrical stimulation of exposed sciatic nerve $(10 \mathrm{~V}, 50 \mathrm{~Hz}, 0.1 \mathrm{~ms})$. Finally sciatic nerve stimulation-evoked EPPs was digitized by PowerLab 8/30 Data Acquisition System with LabChart 7 (AD Instruments), and stored on computer for analyzing the amplitude of EPPs. In the gastrocnemius muscle from each mouse, 6-8 sites were selected for EPPs recording.

\section{Immunohistochemistry of the NMJs}

\section{(Tu et al., 2017)}

After recording of the EPP in-situ, mouse gastrocnemius muscle was quickly isolated for postfixing with $4 \%$ paraformaldehyde for $15 \mathrm{~min}$, and subsequently incubating with $0.1 \mathrm{M}$ glycine for $15 \mathrm{~min}$. To facilitate probe penetrations into NMJs, The gastrocnemius muscle was divided into 8-10 small longitudinal segments, and then permeabilized in $-20^{\circ} \mathrm{C}$ methanol for 10 min. After blocking with PBS containing 0.5\% Triton and 1\% BSA for $1 \mathrm{~h}$, small longitudinal segments of the gastrocnemius 

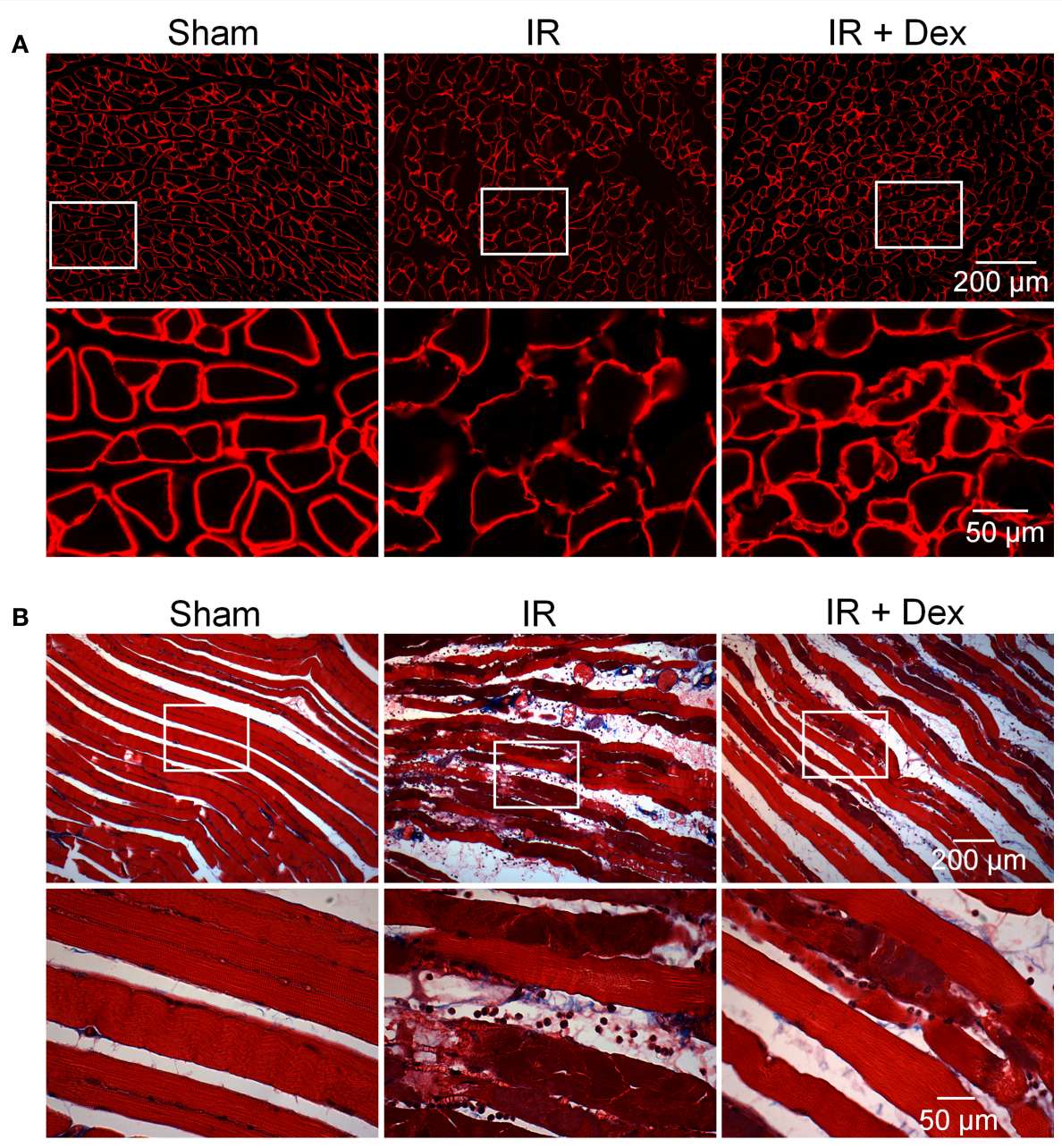

C

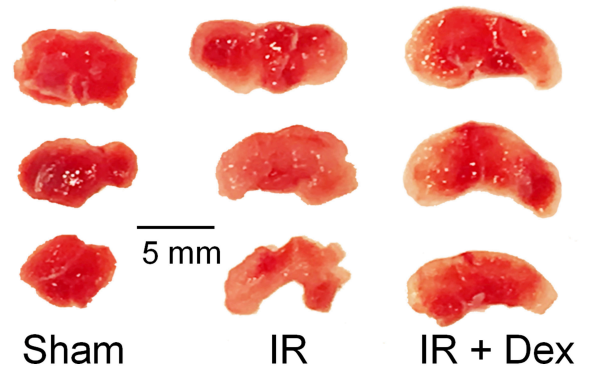

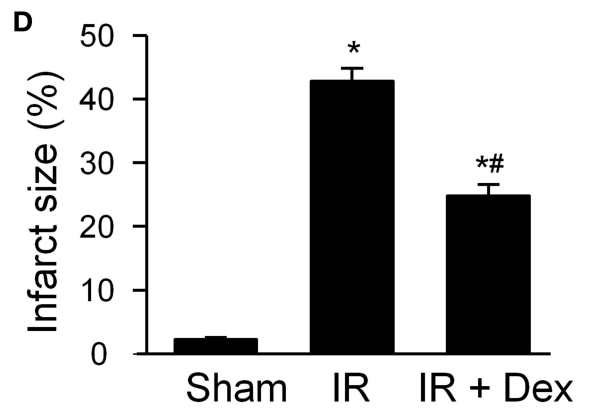

FIGURE 1 | The morphology of gastrocnemius muscles in all experimental groups. (A), histology in the cross section of gastrocnemius muscles, measured by laminin staining (a marker for muscle sarcolemma). (B), histology in the longitudinal section of gastrocnemius muscles, measured by Masson's trichrome staining (C,D), representative and summary data for infarct size in gastrocnemius muscles. Data are mean \pm SEM, $n=5$ mice in each group. ${ }^{\star} P<0.05$ vs. sham; $\# P<$ 0.05 vs. IR.

muscle were incubated overnight at $4^{\circ} \mathrm{C}$ in a cocktail of primary antibodies, including mouse anti-neurofilament 200 (SigmaAldrich, St. Louis, MO) and rabbit anti-synaptophysin (Thermo Fisher Scientific, Waltham, MA) antibodies for axon and nerve terminal labeling. Then muscle segments were incubated overnight at $4^{\circ} \mathrm{C}$ with Alexa Fluor ${ }^{\circledR} 594$ labeled donkey antimouse (Thermo Fisher Scientific, Waltham, MA) and antirabbit (Thermo Fisher Scientific, Waltham, MA) IgGs, and Alexa
Fluor ${ }^{\circledR} 488$ labeled $\alpha$-Bungarotoxin ( $\alpha$-BTX, Thermo Fisher Scientific, Waltham, MA). Finally, images of muscle segments mounted on glass slides were captured using a Leica fluorescent microscope (Leica DMR, Leica Microsystems Inc., Buffalo Grove, IL) to analyze immunohistochemically labeled NMJs including motor nerve terminals and $\mathrm{AAChR}$ clusters.

In each muscle segment, five different regions were selected to obtain Z-stack images of the NMJ. All analyses were done by 


\section{A Raw recordings}

\section{Sciatic nerve stimulation}

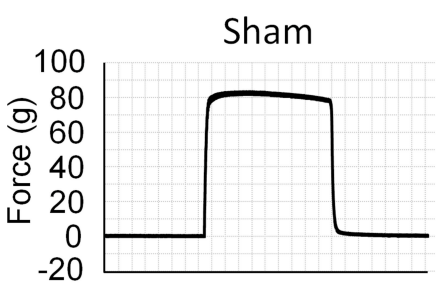

IR
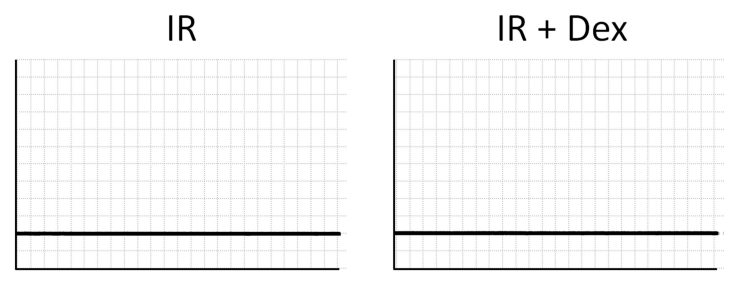

Muscle stimulation at 50V
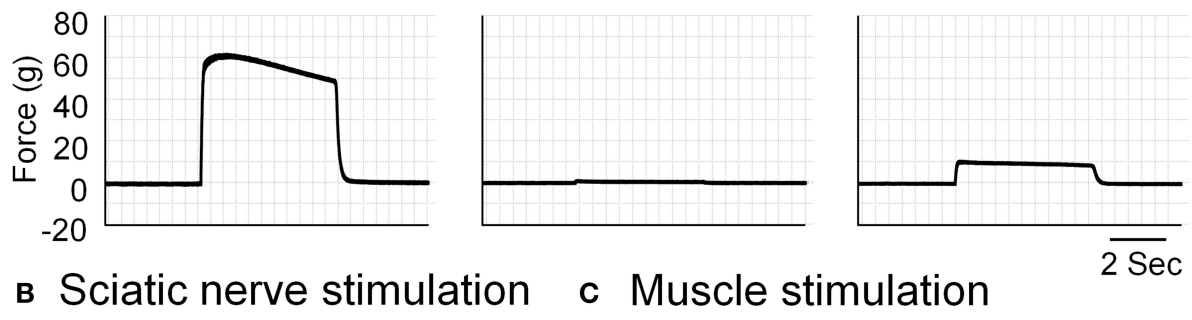

c Muscle stimulation
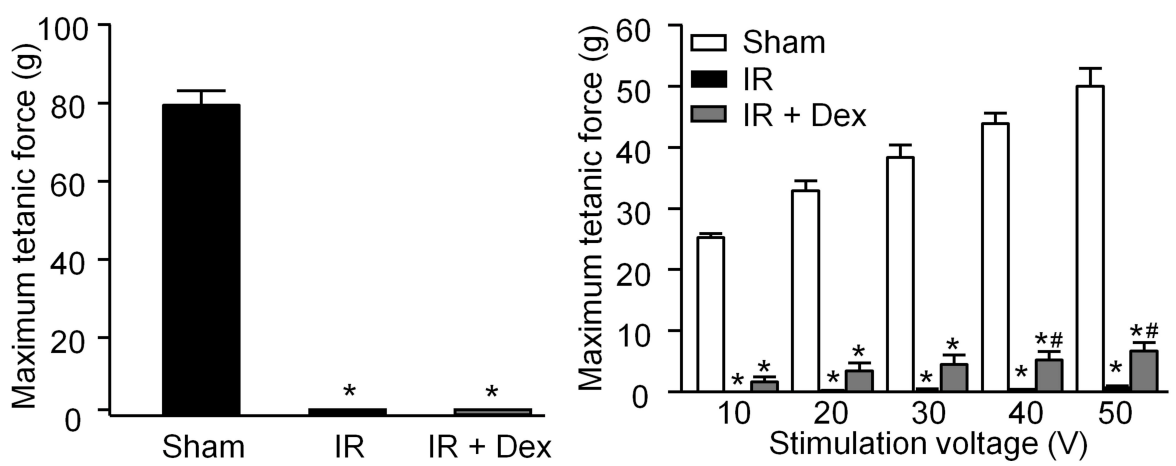

FIGURE 2 | Representative (A) and summary (B,C) data for in situ gastrocnemius muscle tetanic contraction elicited by sciatic nerve stimulation or direct muscle stimulation in all experimental groups. Data are mean $\pm \mathrm{SEM}, n=5$ mice in each group. ${ }^{\star} P<0.05$ vs. sham; $\# P<0.05$ vs. IR.

ImageJ software (NIH Image) on en-face NMJs. In the NMJ, the percentage of motor nerve innervation to $\mathrm{nAChR}$ clusters was quantified by measurements of nerve terminal sizes labeled with neurofilament and synaptophysin. The endplate with or without labeling for neurofilament and synaptophysin was defined as an innervated or denervated endplate, respectively. The $\mathrm{nAChR}$ areas in NMJs labeled with $\alpha$-BTX were used to calculate the area per fragment, the $n A C h R$ area per $n A C h R$ cluster, and the number of discrete fragments per nAChR cluster. A fragmented $\mathrm{nAChR}$ cluster was defined when the number of discrete fragments per $\mathrm{nAChR}$ cluster $\geq 5$.

\section{Protein Expression of II-1 $\beta$ and TNF $\alpha$ in Gastrocnemius Muscles}

Gastrocnemius muscles from five sham, five tourniquet-induced IR, and five tourniquet-induced IR + Dex mice were rapidly removed and stored at $-80^{\circ} \mathrm{C}$ until analyzed. A lysing buffer (10 mM Tris, 1 mM EDTA, $150 \mathrm{mM} \mathrm{NaCl}, 1 \%$ SDS, 1 mM PMSF; $\mathrm{pH}$ 7.4) plus protease inhibitor cocktail (100 $\mu \mathrm{l} / \mathrm{ml}, \mathrm{P} 2714$, Sigma-Aldrich, St. Louis, MO) was added into the muscle tissue for homogenization. Homogenized muscle tissue was centrifuged at $12,000 \mathrm{~g}$ for $20 \mathrm{~min}$ at $4^{\circ} \mathrm{C}$. Total protein concentration in the supernatant was measured by a bicinchoninic acid protein assay kit (Thermo Fisher Scientific, Waltham, MA). Same volume of the loading buffer was added into protein samples to $\mathrm{mix}$ and heat for $5 \mathrm{~min}$ at $100^{\circ} \mathrm{C}$. Protein samples (40 $\mathrm{\mu g} /$ well) loaded in the stacking gel were separated on a $12 \%$ sodium dodecyl sulfate (SDS)-polyacrylamide running gel. Proteins of these samples were electrophoretically transferred at $200 \mathrm{~mA}$ for $3 \mathrm{~h}$ onto PVDF membrane (EMD Millipore, Billerica, MA). The membrane was blocked with 5\% non-fat milk for $1 \mathrm{~h}$. Then the membrane was probed with rabbit anti TNF- $\alpha$ antibody (Cell Signaling Technology, Beverly, MA), mouse anti IL-1 $\beta$ antibody (Cell Signaling Technology, Beverly, MA), or mouse anti $\beta$-actin antibody (Santa Cruz Biotechnology, Dallas, TX) overnight at $4^{\circ} \mathrm{C}$. After washing by PBS, the membrane was incubated with peroxidase-conjugated goat anti-rabbit IgG (Thermo Fisher Scientific, Waltham, MA), or goat anti-mouse IgG (Thermo Fisher Scientific, Waltham, MA) for $1 \mathrm{~h}$. The signal was developed by enhanced chemiluminescence substrate (Thermo Fisher Scientific, Waltham, MA), and captured by UVP bioimaging system (UVP, Upland, CA). 


\section{A Raw image}
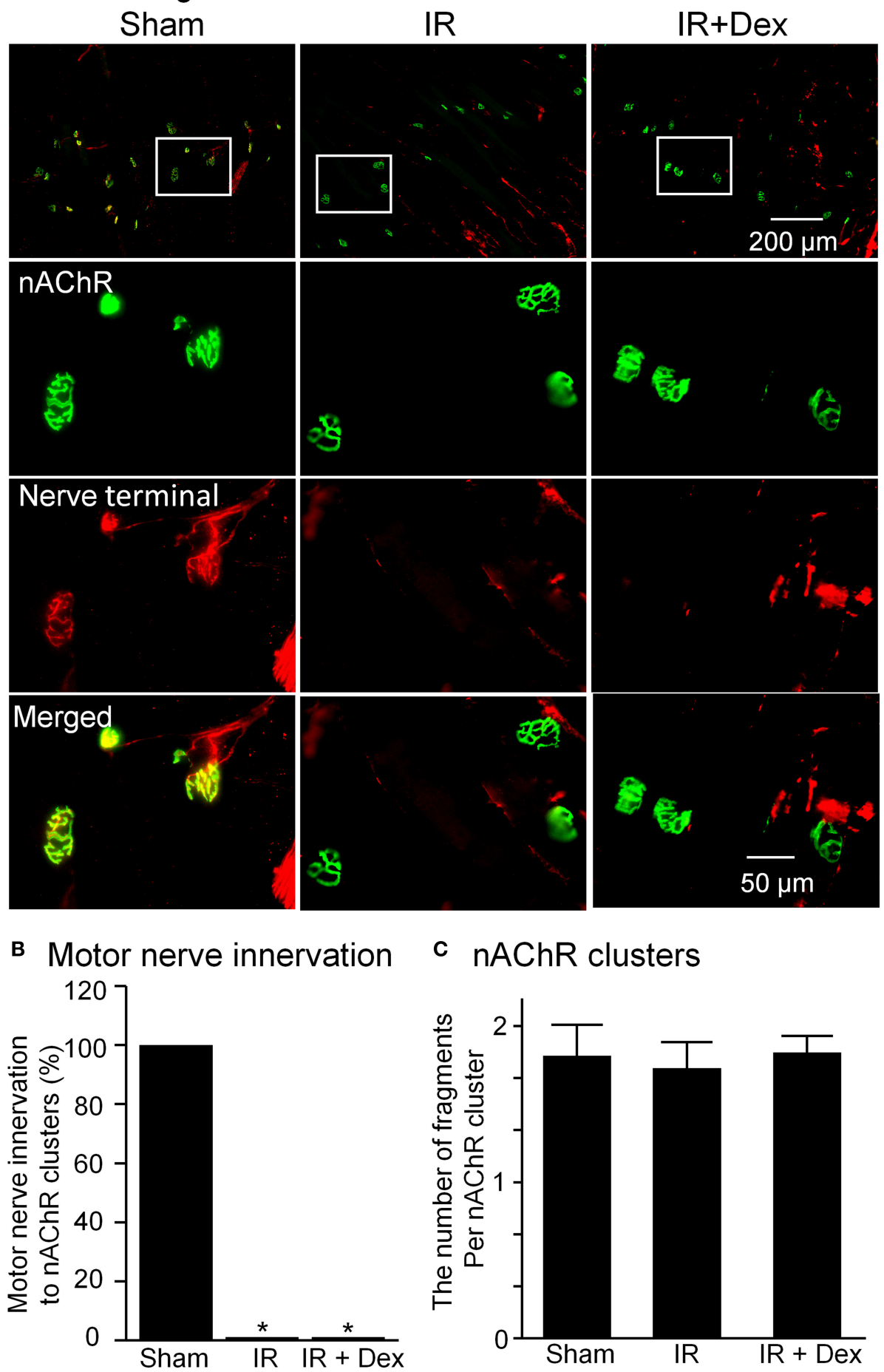

FIGURE 3 | The morphology of NMJs including motor nerve terminals and nAChRs located on gastrocnemius muscles from all experimental groups. (A), representative images of NMJs. Synaptophysin and neurofilament 200 (red color) and $\alpha$-bungarotoxin (BTX, green color) were used to label motor nerve terminals and nAChR clusters in NMJs. (B,C), mean data for motor nerve innervation to nAChR clusters and the number of fragments per nAChR cluster. Data are mean \pm SEM, $n=5$ mice in each group. ${ }^{\star} P<0.05$ vs. sham. 


\section{Statistical Analysis}

All data are presented as means \pm SE. SigmaPlot 12 was used for data analysis. A one-way ANOVA with post hoc Bonferroni test was used to determine statistical significance for multi group comparison. A Chi-Square test was performed for percentage of nerve innervation in the NMJ. Normal distribution of data was confirmed with Kolmogorov-Smirov test and equal variance with Levene's test. Statistical significance was accepted when $p<0.05$.

\section{RESULTS}

\section{Effects of Dex on Acute IR-Induced Morphological and Functional Changes in Gastrocnemius Muscles}

Acute IR-induced gastrocnemius muscle injuries were shown in Figure 1. An obvious breakage of muscle fiber was observed in tourniquet-induced IR group of mice, revealed by laminin staining of gastrocnemius muscles in cross-section (Figure 1A). Tourniquet-induced IR caused undulating muscle cell borders, irregular spacing of muscle striations, and disruption of muscle fibers, evidenced by Massion's trichrome staining of gastrocnemius muscles in longitudinal-section (Figure 1B). Additionally, infarct size of the skeletal muscle was measured by triphenyl tetrazolium chloride assay. Tourniquet-induced IR also markedly resulted in infarction in gastrocnemius muscles $(42.8 \pm 2.4 \%)$, compared to that in sham group of mice $(0 \%$, $p<0.05$, Figures 1C,D). Dex treatment (1 mg/kg, i.p.) at the beginning of reperfusion significantly attenuated, but did not abolish, acute IR-induced gastrocnemius muscle injuries including broken muscle sarcolemmata, disrupted muscle fibers, and muscle infarct size, measured by above three methods (Figure 1).

Gastrocnemius muscle contraction was measured by sciatic nerve stimulation or direct muscle stimulation, which can determine if IR-induced skeletal muscle contractile dysfunction is caused by muscle injury alone and/or by nerve-muscle transmission failure (Chervu et al., 1989; Eastlack et al., 2004). In tourniquet-induced IR group, both sciatic nerve stimulation and direct muscle stimulation did not evoke gastrocnemius muscle contraction (Figure 2). Dex treatment improved direct muscle stimulation-induced muscle contraction in IR-injured gastrocnemius muscles ( $P<0.05$ vs. IR group, Figure 2C), whereas it did not affect sciatic nerve stimulation-induced gastrocnemius muscle contraction (Figure 2B).

\section{Effects of Dex on Structural and Functional Alterations in NMJs}

In Figure 3, motor nerve terminals labeled by neurofilament and synaptophsin and nAChR clusters labeled by BTX were investigated. As a major feature of NMJs, a specific synapse is normally formed by the presynaptic membrane (motor nerve terminals) and postsynaptic membrane (nAChR clusters), which can transmit the signal from motor neurons to skeletal muscles (Figure 3A). In IR group, the gastrocnemius muscle was totally denervated by motor nerve terminals in all NMJs $(0 \%$ motor nerve innervation to $\mathrm{nAChR}$ clusters, $p<0.05$ vs. sham). Compared to sham mice, the structure of nAChR clusters did not show significant alterations, and the number of fragments per nAChR cluster had no markedly changes ( $1.65 \pm 0.13$ vs. sham $1.80 \pm 0.21$, Figure $3 C)$. Treatment with Dex $(1 \mathrm{mg} / \mathrm{kg}) \mathrm{did}$ not blunt IR-induced disappearance of motor nerve terminals in NMJs from the gastrocnemius muscle (Figure 3).

As noted above, electrical signals are usually transmitted from motor neurons to skeletal muscles through the NMJ (including motor nerve terminals and nAChR clusters), which can trigger skeletal muscle contraction. Recording of sciatic nerve-stimulated EPPs was used to NMJ function in the gastrocnemius muscle (Figure 4). In sham animals, sciatic nerve stimulation evokes regular, steady EPPs and the amplitude of EPPs was $29.9 \pm 1.4 \mathrm{mV}$. In the IR group, sciatic nerve-stimulated EPPs were not detectable in all NMJs (Figure 4). When Dex (1 $\mathrm{mg} / \mathrm{kg}$ ) was administered at the beginning of reperfusion, IRinduced disappearance of sciatic nerve-stimulated EPPS was not improved (Figure 4).

\section{Effects of Dex on Inflammatory Cytokines in IR Gastrocnemius Muscles}

In the sham group, there was no obvious leukocyte infiltration and expression of IL- $1 \beta$ and $\mathrm{TNF} \alpha$ (2 proinflammatory cytokines) kept at a low level in the gastrocnemius muscle (Figure 5). However, after $3 \mathrm{~h}$ of tourniquet and $24 \mathrm{~h}$ of

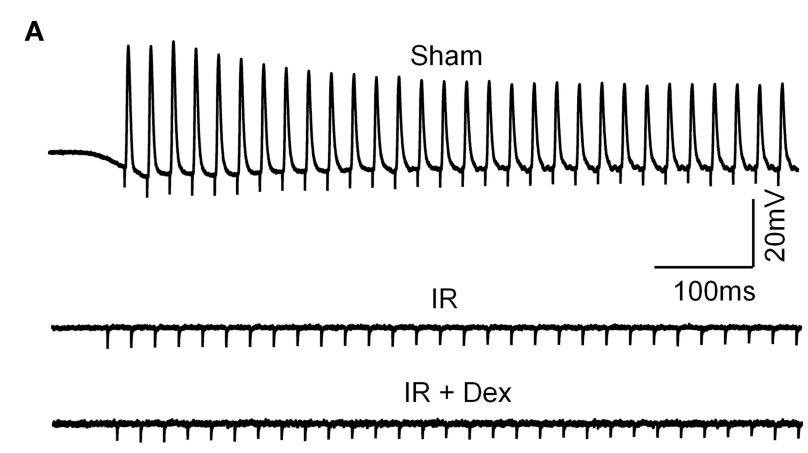

B

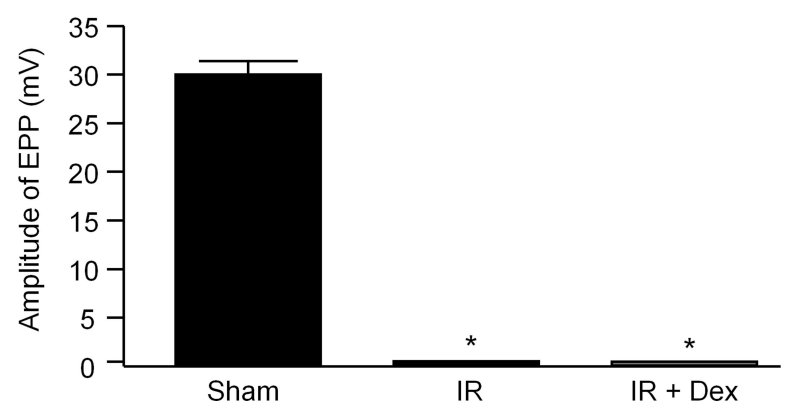

FIGURE 4 | Representative (A) and summary (B) data for sciatic nerve-stimulated endplate potentials (EPPS) recorded in gastrocnemius muscles from all experimental groups. Data are mean \pm SEM, $n=5$ mice in each group. ${ }^{\star} P<0.05$ vs. sham. 
reperfusion, a large number of leukocytes, including neutrophils, lymphocytes, and monocytes were infiltrated into the IRinjured gastrocnemius muscle. Likewise, expression of IL-1 $\beta$ and $\mathrm{TNF} \alpha$ significantly increased in the IR-injured gastrocnemius muscle. When Dex $(1 \mathrm{mg} / \mathrm{kg})$ was administered at the beginning of reperfusion, IR-induced leukocyte infiltration and overexpression of IL-1 $\beta$ and TNF $\alpha$ were markedly decreased $(p<0.05$ vs. IR group, Figure 5).

\section{DISCUSSION}

In the present study, we mimicked the clinical tourniquet use to reproduce the critical limb syndrome in a mouse model subjected to $3 \mathrm{~h}$ of tourniquet and $24 \mathrm{~h}$ of reperfusion. In this mouse model, tourniquet-induced IR caused not only the morphological and functional damage of skeletal muscles and NMJs, but also the leukocyte infiltration and over-production of inflammatory cytokines in skeletal muscles. Treatment of Dex at the beginning of reperfusion significantly inhibited IR-induced leukocyte infiltration and overexpression of IL- $1 \beta$ and TNF $\alpha$ in gastrocnemius muscles. This anti-inflammatory drug also blunted acute IR-induced gastrocnemius muscle injuries and ameliorated direct muscle stimulation-evoked muscle contraction in IRinjured gastrocnemius muscles. However, Dex did not avert IR-induced disappearance of motor nerve terminals in NMJs from gastrocnemius muscles, and did not improve sciatic nerve-stimulated EPPs and muscle contraction in IR-injured gastrocnemius muscles. These results suggest that treatment of Dex at the beginning of reperfusion, through inhibiting inflammatory cytokines, alleviate acute IR-induced structural

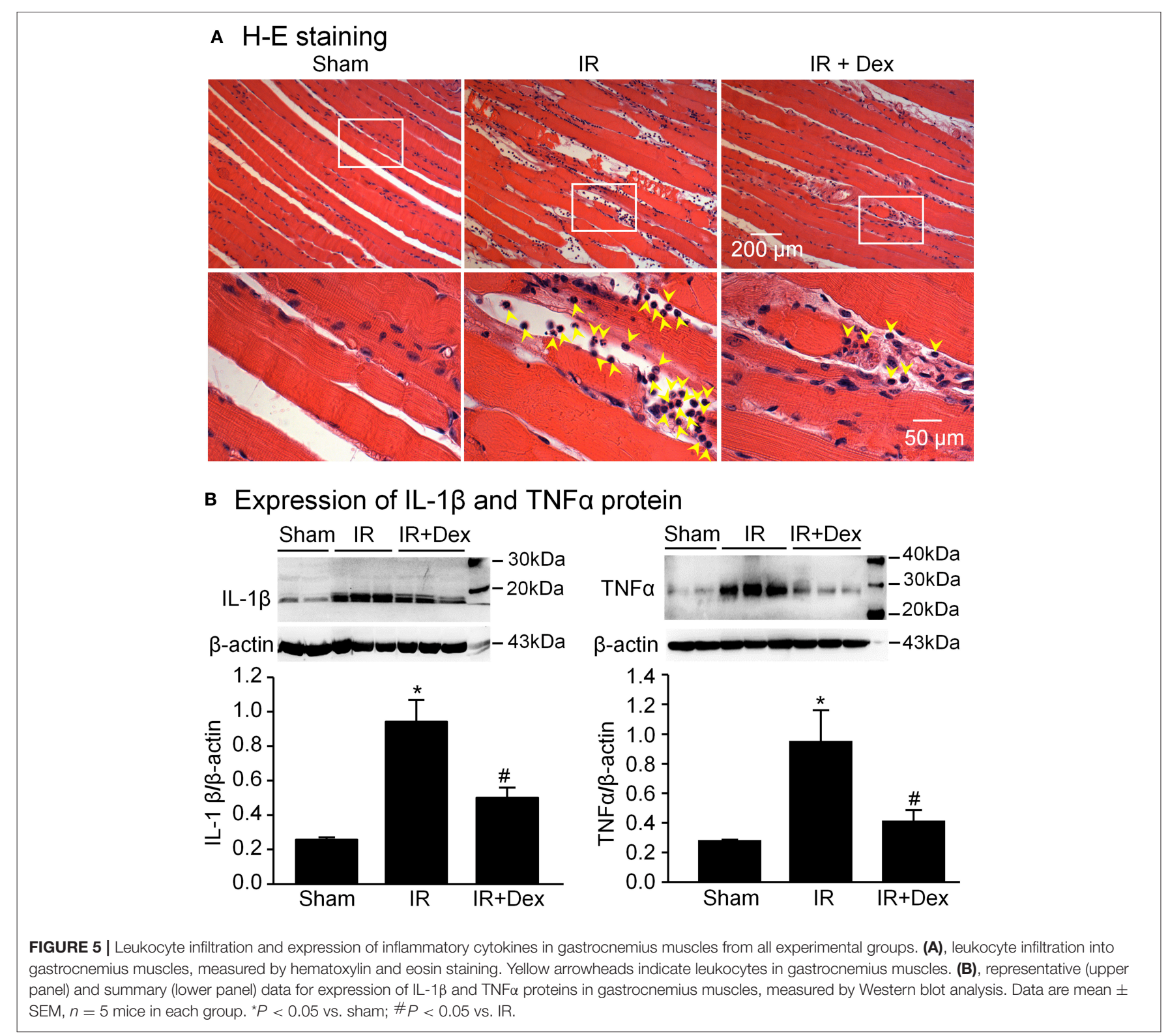


and functional impairments of gastrocnemius muscles but not NMJs.

There is a long history for tourniquet use in first-line treatment of severe limb hemorrhage. Although limited clinical evidence exists about complications associated with the use of this device due to the impossibility of distinguishing between tourniquet-induced and original hemorrhage-induced complications, concerns about tourniquet-induced nerve palsy, skeletal muscle injury, and amputation have led to the limitation of tourniquet use, suggesting as a last option after other hemorrhage control interventions fail (Clasper et al., 2009; Kue et al., 2015). Therefore, finding effective therapy for tourniquetinduced IR injuries can resolve the limitations of tourniquet use, and significantly improve outcomes and quality of life for this large group of patients.

Glucocorticoids such as Dex are widely used in clinical patients as potent anti-inflammatory drugs (Urbanska et al., 2014; Rodriguez et al., 2017). A previous study reported that the intramuscular administration of Dex $3 \mathrm{~h}$ prior to tourniquet successfully attenuated IR-induced muscle contractile dysfunction seen in rat extensor digitalis longus muscles following tourniquet-induced $3 \mathrm{~h}$ of ischemia and $24 \mathrm{~h}$ of reperfusion (Chen et al., 1996). As everyone knows, however, it is unrealistic to expect clinical pretreatment of drugs for tourniquet-induced IR injuries because extremity hemorrhage and tourniquet use are not predicted. To determine the clinical relevance of drugs, therefore, treatment of drugs at the beginning of reperfusion is an optimal timing for investigation of drugs' effects on tourniquet-induced IR injuries. In the present study, treatment of Dex at the beginning of reperfusion attenuated acute IR-induced gastrocnemius muscle injuries and improved direct muscle stimulation-evoked muscle contraction in IRinjured gastrocnemius muscles. It has been shown that Dex binding to the glucocorticoid receptor inhibits inflammatory cytokines through inhibition of NF- $\mathrm{B}$ in some inflammatory conditions (Uddin et al., 2011). Our current study found that Dex significantly inhibited IR-induced overexpression of IL- $1 \beta$ and TNF $\alpha$ in gastrocnemius muscles. Analyzing above results, we thought that treatment of Dex at the beginning of reperfusion could improve the structure and function of skeletal muscles experienced $3 \mathrm{~h}$ of tourniquet and $24 \mathrm{~h}$ of reperfusion, through inhibition of inflammatory cytokine production. Nevertheless, treatment of Dex did not affect the structure and function of NMJs in IR-injured gastrocnemius muscles. It is possible that other cellular and molecular mechanisms (such as overproduction of reactive oxygen species and dysfunction of the endogenous antioxidative system) are responsible for acute IR-induced morphological and functional

\section{REFERENCES}

Aho, K., Sainio, K., Kianta, M., and Varpanen, E. (1983). Pneumatic tourniquet paralysis. case report. J. Bone Joint Surg. Br. 65, 441-443. doi: 10.1302/0301-620X.65B4.6874716

Arango, D. G., and Descoteaux, A. (2014). Macrophage cytokines: involvement in immunity and infectious impairments of NMJs in the skeletal muscle, because G93A mutation of superoxide dismutase significantly increases the denervation of motor nerve terminals during skeletal muscle tourniquet-induced IR (David et al., 2007).

Inflammatory cytokines are mainly produced by activated leukocytes, including neutrophils, monocytes, and lymphocytes (Arango and Descoteaux, 2014). In the current study, tourniquetinduced IR caused the leukocyte infiltration into IR-injured gastrocnemius muscles. Treatment of Dex obviously blunted IR-induced leukocyte infiltration, which suggests that Dex decreased expression of IL-1 $\beta$ and TNF $\alpha$ in IR-injured gastrocnemius muscles, through inhibiting leukocyte activation and infiltration. It is possible that the skeletal muscle itself is also involved in IR-induced overexpression of IL-1 $\beta$ and TNF $\alpha$, because the skeletal muscle is thought to be an endocrine organ for muscle-derived cytokine production (Pedersen and Febbraio, 2008; Borge et al., 2009; Arango and Descoteaux, 2014).

Our current study did not identify the contribution of each cytokine (IL-1 $\beta$ and TNF $\alpha$ ) to tourniquet-induced IR injuries. Additionally, clinical use of Dex is hampered by its severe systemic side effects (Curtis et al., 2006; Fardet et al., 2007; Hanaoka et al., 2012). Therefore, the effects of IL-1 receptor antagonist (IL-1RA) and TNF $\alpha$ inhibitors (etanercept, infliximab, adalimumab, etc.) on tourniquet-induced IR injuries will be investigated in future study to discover the optimum clinical therapeutics.

In conclusion, tourniquet-induced IR results in morphological and functional impairments in mouse skeletal muscles and NMJs. Treatment of Dex at the beginning of reperfusion, through inhibiting inflammatory cytokines, protects the skeletal muscle from acute IR injuries, whereas it does not ameliorate the structure and function of NMJs in IR-injured skeletal muscles.

\section{AUTHOR CONTRIBUTIONS}

RC, HT, DZ, and Y-LL designed and performed experiments, analyzed data, and prepared the manuscript. RC, AB, RM, MW, and Y-LL wrote, edited, and revised the manuscript. All authors approved the version to be published.

\section{FUNDING}

This study was supported by the Assistant Secretary of Defense for Health Affairs, USA, 393 through the Defense Medical Research and Development Program under Award No. 394 W81XWH-17-1-0037 (to Y-LL). 
freedom: effect on hemorrhage control and outcomes. J. Trauma 64, S28-S37. doi: 10.1097/TA.0b013e318160937e

Borge, B. A., Kalland, K. H., Olsen, S., Bletsa, A., Berggreen, E., and Wiig, H. (2009). Cytokines are produced locally by myocytes in rat skeletal muscle during endotoxemia. Am. J. Physiol. Heart Circ. Physiol. 296, H735-H744. doi: 10.1152/ajpheart.01309.2008

Center for Disease Control and Prevention (2017). Estimated Number of Extremities Visited to Ed and Cost of Injury Reports. Available online at: https:// wisqars.cdc.gov:8443/costT/

Chen, G. Y., and Nunez, G. (2010). Sterile inflammation: sensing and reacting to damage. Nat. Rev. Immunol. 10, 826-837. doi: 10.1038/nri2873

Chen, L. E., Silver, W. P., Seaber, A. V., Korompilias, A. V., and Urbaniak, J. R. (1996). Effects of dexamethasone on the contractile function of reperfused skeletal muscle. Microsurgery 17, 313-320. doi: 10.1002/(SICI) 1098-2752(1996)17:6<313::AID-MICR5>3.0.CO;2-I

Chervu, A., Moore, W. S., Homsher, E., and Quinones-Baldrich, W. J. (1989). Differential recovery of skeletal muscle and peripheral nerve function after ischemia and reperfusion. J. Surg. Res. 47, 12-19. doi: 10.1016/0022-4804(89)90041-3

Clasper, J. C., Brown, K. V., and Hill, P. (2009). Limb complications following pre-hospital tourniquet use. J. R. Army Med. Corps 155, 200-202. doi: 10.1136/jramc-155-03-06

Clementsen, T., and Reikeras, O. (2008). Cytokine patterns after tourniquetinduced skeletal muscle ischaemia reperfusion in total knee replacement. Scand. J. Clin. Lab. Invest. 68, 154-159. doi: 10.1080/00365510701528587

Curtis, J. R., Westfall, A. O., Allison, J., Bijlsma, J. W., Freeman, A., George, V., et al. (2006). Population-based assessment of adverse events associated with longterm glucocorticoid use. Arthritis Rheum. 55, 420-426. doi: 10.1002/art.21984

David, G., Nguyen, K., and Barrett, E. F. (2007). Early vulnerability to ischemia/reperfusion injury in motor terminals innervating fast muscles of SOD1-G93A mice. Exp. Neurol. 204, 411-420. doi: $10.1016 /$ j.expneurol.2006.12.021

Eastlack, R. K., Groppo, E. R., Hargens, A. R., and Pedowitz, R. A. (2004). Ischemic-preconditioning does not prevent neuromuscular dysfunction after ischemia-reperfusion injury. J. Orthop. Res. 22, 918-923. doi: 10.1016/j.orthres.2003.10.015

Eltzschig, H. K., and Eckle, T. (2011). Ischemia and reperfusion-from mechanism to translation. Nat. Med. 17, 1391-1401. doi: 10.1038/nm.2507

Fardet, L., Kassar, A., Cabane, J., and Flahault, A. (2007). Corticosteroid-induced adverse events in adults: frequency, screening and prevention. Drug Saf. 30, 861-881. doi: 10.2165/00002018-200730100-00005

Fish, J. S., McKee, N. H., Pynn, B. R., Kuzon, W. M. Jr., and Plyley, M. J. (1989). Isometric contractile function recovery following tourniquet ischemia. J. Surg. Res. 47, 365-370. doi: 10.1016/0022-4804(89)90149-2

Gillani, S., Cao, J., Suzuki, T., and Hak, D. J. (2012). The effect of ischemia reperfusion injury on skeletal muscle. Injury 43, 670-675. doi: 10.1016/j.injury.2011.03.008

Halladin, N. L., Zahle, F. V., Rosenberg, J., and Gogenur, I. (2014). Interventions to reduce tourniquet-related ischaemic damage in orthopaedic surgery: a qualitative systematic review of randomised trials. Anaesthesia 69, 1033-1050. doi: 10.1111/anae.12664

Hanaoka, B. Y., Peterson, C. A., Horbinski, C., and Crofford, L. J. (2012). Implications of glucocorticoid therapy in idiopathic inflammatory myopathies. Nat. Rev. Rheumatol. 8, 448-457. doi: 10.1038/nrrheum.2012.85

Inaba, K., Siboni, S., Resnick, S., Zhu, J., Wong, M. D., Haltmeier, T., et al. (2015). Tourniquet use for civilian extremity trauma. J. Trauma Acute Care Surg. 79, 232-237. doi: 10.1097/TA.0000000000000747

Kam, P. C., Kavanagh, R., and Yoong, F. F. (2001). The arterial tourniquet: pathophysiological consequences and anaesthetic implications. Anaesthesia 56, 534-545. doi: 10.1046/j.1365-2044.2001.01982.x

Kragh, J. F. Jr., Swan, K. G., Smith, D. C., Mabry, R. L., and Blackbourne, L. H. (2012). Historical review of emergency tourniquet use to stop bleeding. Am. J. Surg. 203, 242-252. doi: 10.1016/j.amjsurg.2011.01.028

Kue, R. C., Temin, E. S., Weiner, S. G., Gates, J., Coleman, M. H., Fisher, J., et al. (2015). Tourniquet use in a civilian emergency medical services setting: a descriptive analysis of the boston EMS experience. Prehosp. Emerg. Care 19, 399-404. doi: 10.3109/10903127.2014.995842
Lotfi, S., Patel, A. S., Mattock, K., Egginton, S., Smith, A., and Modarai, B. (2013). Towards a more relevant hind limb model of muscle ischaemia. Atherosclerosis 227, 1-8. doi: 10.1016/j.atherosclerosis.2012.10.060

Mabry, R. L. (2006). Tourniquet use on the battlefield. Mil. Med. 171, 352-356. doi: 10.7205/MILMED.171.5.352

Mohler, L. R., Pedowitz, R. A., Lopez, M. A., and Gershuni, D. H. (1999). Effects of tourniquet compression on neuromuscular function. Clin. Orthop. Relat. Res. 213-220. doi: 10.1097/00003086-199902000-00024

Noordin, S., McEwen, J. A., Kragh, J. F. Jr., Eisen, A., and Masri, B. A. (2009). Surgical tourniquets in orthopaedics. J. Bone Joint Surg. Am. 91, 2958-2967. doi: 10.2106/JBJS.I.00634

Ode, G., Studnek, J., Seymour, R., Bosse, M. J., and Hsu, J. R (2015). Emergency tourniquets for civilians: can military lessons in extremity hemorrhage be translated?. J. Trauma Acute Care Surg. 79, 586-591. doi: 10.1097/TA.0000000000000815

Pedersen, B. K., and Febbraio, M. A. (2008). Muscle as an endocrine organ: focus on muscle-derived interleukin-6. Physiol. Rev. 88, 1379-1406. doi: 10.1152/physrev.90100.2007

Rodriguez, V. J., Rodriguez, V. L., and Guzman, N. M. (2017). Pharmaceutical technology can turn a traditional drug, dexamethasone into a first-line ocular medicine. a global perspective and future trends. Int. J. Pharm. 516, 342-351. doi: 10.1016/j.ijpharm.2016.11.053

Schoen, M., Rotter, R., Gierer, P., Gradl, G., Strauss, U., Jonas, L., et al. (2007). Ischemic preconditioning prevents skeletal muscle tissue injury, but not nerve lesion upon tourniquet-induced ischemia. J. Trauma 63, 788-797. doi: 10.1097/01.ta.0000240440.85673.fc

Schuld, A., Kraus, T., Haack, M., Hinze-Selch, D., Zobel, A. W., Holsboer, F., et al. (2001). Effects of dexamethasone on cytokine plasma levels and white blood cell counts in depressed patients. Psychoneuroendocrinology 26, 65-76. doi: 10.1016/S0306-4530(00)00039-1

Schwiebert, L. M., Beck, L. A., Stellato, C., Bickel, C. A., Bochner, B. S., and Schleimer, R. P. (1996). Glucocorticosteroid inhibition of cytokine production: relevance to antiallergic actions. J. Allergy Clin. Immunol. 97, 143-152. doi: 10.1016/S0091-6749(96)80214-4

Seekamp, A., Warren, J. S., Remick, D. G., Till, G. O., and Ward, P. A. (1993). Requirements for tumor necrosis factor-alpha and interleukin-1 in limb ischemia/reperfusion injury and associated lung injury. Am. J. Pathol. 143, 453-463.

Smits, H. H., Grunberg, K., Derijk, R. H., Sterk, P. J., and Hiemstra, P. S. (1998). Cytokine release and its modulation by dexamethasone in whole blood following exercise. Clin. Exp. Immunol. 111, 463-468. doi: 10.1046/j.1365-2249.1998.00482.x

Tai, T. W., Lin, C. J., Jou, I. M., Chang, C. W., Lai, K. A., and Yang, C. Y. (2011). Tourniquet use in total knee arthroplasty: a meta-analysis. Knee Surg. Sports Traumatol. Arthrosc. 19, 1121-1130. doi: 10.1007/s00167-010$1342-7$

Tran, T. P., Tu, H., Liu, J., Muelleman, R. L., and Li, Y. L. (2012). Mitochondria-derived superoxide links to tourniquet-induced apoptosis in mouse skeletal muscle. PLOS ONE 7:e43410. doi: 10.1371/journal.pone. 0043410

Tran, T. P., Tu, H., Pipinos, I. I., Muelleman, R. L., Albadawi, H., and Li, Y. L. (2011). Tourniquet-induced acute ischemia-reperfusion injury in mouse skeletal muscles: Involvement of superoxide. Eur. J. Pharmacol. 650, 328-334. doi: 10.1016/j.ejphar.2010.10.037

Tu, H., Zhang, D., Corrick, R. M., Muelleman, R. L., Wadman, M. C., and Li, Y. L. (2017). Morphological regeneration and functional recovery of neuromuscular junctions after tourniquet-induced injuries in mouse hindlimb. Front. Physiol. 8:207. doi: $10.3389 /$ fphys.2017.00207

Uddin, M. N., Siddiq, A., Oettinger, C. W., and D'Souza, M. J. (2011). Potentiation of pro-inflammatory cytokine suppression and survival by microencapsulated dexamethasone in the treatment of experimental sepsis. J. Drug Target 19, 752-760. doi: 10.3109/1061186X.2011.561856

Urbanska, J., Karewicz, A., and Nowakowska, M. (2014). Polymeric delivery systems for dexamethasone. Life Sci. 96, 1-6. doi: 10.1016/j.lfs.2013. 12.020

Vignaud, A., Hourde, C., Medja, F., Agbulut, O., Butler-Browne, G., and Ferry, A. (2010). Impaired skeletal muscle repair after ischemia-reperfusion 
injury in mice. J. Biomed. Biotechnol. 2010:724914. doi: 10.1155/2010/ 724914

Walters, T. J., Kragh, J. F., and Baer, D. G. (2008). Influence of fibertype composition on recovery from tourniquet-induced skeletal muscle ischemia-reperfusion injury. Appl. Physiol. Nutr. Metab. 33, 272-281. doi: 10.1139/H07-180

Welling, D. R., McKay, P. L., Rasmussen, T. E., and Rich, N. M. (2012). A brief history of the tourniquet. J. Vasc. Surg. 55, 286-290. doi: 10.1016/j.jvs.2011.10.085

Woitaske, M. D., and McCarter, R. J. (1998). Effects of fiber type on ischemiareperfusion injury in mouse skeletal muscle. Plast. Reconstr. Surg. 102, 2052-2063. doi: 10.1097/00006534-199811000-00037

Zhang, D., Wang, D., Pipinos, I. I., Muelleman, R. L., and Li, Y. L. (2017). Dexamethasone promotes long-term functional recovery of neuromuscular junction in a murine model of tourniquet-induced ischaemia-reperfusion. Acta Physiol. 219, 453-464. doi: 10.1111/apha.12737

Conflict of Interest Statement: The authors declare that the research was conducted in the absence of any commercial or financial relationships that could be construed as a potential conflict of interest.

Copyright (c) 2018 Corrick, Tu, Zhang, Barksdale, Muelleman, Wadman and Li. This is an open-access article distributed under the terms of the Creative Commons Attribution License (CC BY). The use, distribution or reproduction in other forums is permitted, provided the original author(s) and the copyright owner are credited and that the original publication in this journal is cited, in accordance with accepted academic practice. No use, distribution or reproduction is permitted which does not comply with these terms. 